\title{
Knockdown of pseudogene DUXAP8 expression in glioma suppresses tumor cell proliferation
}

\author{
$\mathrm{XU}_{\mathrm{ZHAO}}{ }^{1}$, SHUAI HAO $^{2}$, MINQING WANG $^{1}$, DEGUANG XING $^{1}$ and CHENGWEI WANG $^{1}$ \\ ${ }^{1}$ Department of Neurosurgery, The Second Hospital of Shandong University, Jinan, Shandong 250033; \\ ${ }^{2}$ Department of Neurosurgery, People's Hospital of Juye County, Juye, Shandong 274900, P.R. China
}

Received February 25, 2018; Accepted December 3, 2018

DOI: $10.3892 /$ ol.2019.9994

\begin{abstract}
A large number of pseudogenes as well as long non-coding RNAs (lncRNAs) have been identified as important regulators in human tumors. However, the clinical role and potential functional effects of the double homeobox A pseudogene 8 (DUXAP8) in glioma remains unknown. In the present study, it was revealed that pseudogene DUXAP8 is significantly upregulated in glioma tissues, compared with adjacent normal tissues. Patients with increased DUXAP8 expression were associated with higher Karnofsky Performance Status, advanced World Health Organization grade, poor disease-free survival and overall survival rates of patients with glioma. Furthermore, in vitro assays, Cell-Counting Kit- 8 cell viability and cell colony forming assays demonstrated that reduced DUXAP8 expression significantly suppressed proliferation capacity. Therefore, the results of the present study indicate that pseudogene DUXAP8 is an oncogenic lncRNA and may serve as a potentially prognostic biomarker and novel target of glioma treatment.
\end{abstract}

\section{Introduction}

Glioma is the most malignant and incurable brain tumor with a poor outcome globally (1). Despite advances in standard therapy, including surgical resection, radiotherapy and chemotherapy, the 5-year survival rate remains dismal $(2,3)$. Therefore, identifying novel diagnostic and prognostic makers is essential.

Pseudogenes were initially regarded as non-functional genomic fossils resulting from inactivating gene mutations during evolution (4). Pseudogene-derived RNAs serve multifaceted roles, including post-transcriptional regulation identified as antisense RNAs, endogenous small-interference RNAs and

Correspondence to: $\mathrm{Dr}$ Chengwei Wang, Department of Neurosurgery, The Second Hospital of Shandong University, 247 Beiyuan Road, Jinan, Shandong 250033, P.R. China

E-mail: jzo5119017558@163.com

Key words: glioma, pseudogene, double homeobox A pseudogene 8, tumor prognosis, proliferation competing endogenous RNAs $(5,6)$. Previous studies revealed that peudogenes exhibit multilayered biological functions in multiple cellular processes, including proliferation, migration and invasion, in numerous tumor types $(5,7)$. For instance, small ubiquitin-like modifier 1 pseudogene 3 (SUMO1P3) had a significantly increased expression in gastric cancer, and its expression was significantly associated with tumor size, differentiation, lymphatic metastasis and invasion (8). Increased expression of SUMO1P3 predicts poor prognosis and promotes tumor growth and metastasis in bladder cancer (9). Zinc finger protein 91 pseudogene promotes the migration of BXPC-3-H cells and may be a novel marker for early diagnosis for pancreatic cancer (10). DUXAP8 is identified to act as an oncogene in non-small cell lung cancer (NSCLC) and promotes NSCLC progression (11). In gastric cancer, DUXAP8 could epigenetically suppress the expression of pleckstrin homology domain containing $\mathrm{O} 1$ and enhance proliferation and migration (12). However, the role of pseudogene DUXAP8 in glioma progression remains unknown.

In the present study, it was revealed that pseudogene DUXAP8 is significantly upregulated in glioma tissues. Patients with increased DUXAP8 expression levels demonstrated poor survival rate, implying that DUXAP8 was a prognostic marker for patients with glioma. It was further demonstrated that knockdown of DUXAP8 suppressed proliferation. Therefore, the results of the present study indicated that pseudogene DUXAP8 may be a potential prognostic biomarker and target of glioma treatment.

\section{Materials and methods}

Patient tissue samples. A total of 58 paired of human glioma tissues and adjacent normal tissue sample were collected from patients including 34 males and 23 females (age range, 31-72 years; median, 52.22 years), who were undergoing surgical resection at the Department of Neurosurgery, The Second Hospital of Shandong University (Jinan, China) between January 2011 and December 2014. The adjacent normal brain tissue was defined as $1 \mathrm{~cm}$ away from the lesions. None of the patients receive treatment, including radiation or chemotherapy, prior to surgery. The patients with glioma were classified as World Health Organization (WHO) I, II, III and IV stage, according to a previous report (13). The tissue samples were snap-frozen in liquid nitrogen immediately following 
resection and stored at $-80^{\circ} \mathrm{C}$. The follow-up date was between March 2012 and January 2017. The follow-up date was between the date of the primary surgery and relapse, patient mortality or the late follow-up date prior to mortality. The study was approved by the Ethics Committee of The Second Hospital of Shandong University (Jinan, China). Written informed consent was obtained from all patients in the study.

Cell lines culture. Human glioma U87 (U-87MG Uppsala), U251 (U-251 MG) and H4 cell lines, and normal human astrocyte NHA cells, were purchased from The Institute of Biochemistry and Cell Biology, Chinese Academy of Sciences (Shanghai, China). All of cells were cultured in Dulbecco's modified Eagle's medium (Gibco; Thermo Fisher Scientific, Inc., Waltham, MA, USA) and supplemented with $10 \%$ fetal bovine serum (Gibco; Thermo Fisher Scientific, Inc.) in a humidified incubator at $37^{\circ} \mathrm{C}$ containing $5 \% \mathrm{CO}_{2}$.

Reverse transcription-quantitative polymerase chain reaction $(R T-q P C R)$. Total RNA was extracted from tissue samples using TRIzol ${ }^{\circledR}$ reagent (Takara Biotechnology Co., Ltd., Dalian, China). RNA integrity was analyzed by using a NanoDrop ND-1000 spectrophotometer (NanoDrop Technologies; Thermo Fisher Scientific, Inc.). RNA was reversed transcribed to cDNA with a PrimeScript ${ }^{\circledR}$ RT reagent kit (Takara Biotechnology Co., Ltd.), according to the manufacturer's protocols. The RT-qPCR reaction was performed on a CFX-96 Real-Time PCR system (Bio-Rad Laboratories, Inc., Hercules, CA, USA) with SYBR ${ }^{\circledR}$ Premix Ex Taq ${ }^{\mathrm{TM}}$ II (Takara Biotechnology Co., Ltd.), according to the manufacturer's protocols. The thermocycling conditions were as follows: Denaturation at $95^{\circ} \mathrm{C}$ for $5 \mathrm{~min}$ followed by 35 cycles of denaturation at $95^{\circ} \mathrm{C}$ for $15 \mathrm{sec}$, and annealing/elongation at $60^{\circ} \mathrm{C}$ for $30 \mathrm{sec}$. The results were normalized to the expression of GAPDH. The primer sequences for DUXAP8 (Access number: NR_122113.1) are as follows: DUXAP8-forward: 5'-GAGAAG CAGTGGTGGGTTCC-3', and DUXAP8-reverse: 5'-GAG CAACACAGATGAACCGC-3'. GAPDH-forward: 5'-GGG AGCCAAAAGGGTCAT-3', and GAPDH-reverse: 5'-GAG TCCTTCCACGATACCAA-3'. The mRNA expression fold changes were calculated using the $2^{-\Delta \Delta \mathrm{Cq}}$ methods (14).

Cell transfection. A total of 2 small interfering (si)RNAs targeting DUXAP8 (si-RNA-1, sense: 5'-UUUAGACCCAUU CUCGUAUGGAGGU-3', and antisense: 5'-ACCUCCAUA CGAGAAUGGGUCUAAA-3'; siRNA-2, sense: 5'-CAGCAU ACUUCAAAUUCACAGCAAA-3', and antisense 5'-UUU GCUGUGAAUUUGAAGUAUGCUG-3') and scrambled negative control siRNA (si-NC; 5'-UUCUCCGAACGUGUC ACGUTT-3') were purchased from Invitrogen (Thermo Fisher Scientific, Inc.). The U87 and U251 cells were transfected with si-NC (100 nM), si-RNA-1 (100 nM) or siRNA-2 (100 nM) using Lipofectamine ${ }^{\circledR} 2000$ (Invitrogen; Thermo Fisher Scientific, Inc.), according to the manufacturer's protocols. Following cell transfection for $48 \mathrm{~h}$, the cells were harvested for RT-qPCR analysis of mRNA expression.

Cell Counting Kit-8 (CCK-8) proliferation assay. The transfected cells $\left(3 \times 10^{3}\right.$ cells/well) were seeded in 96-well culture plates and incubated with $10 \mu \mathrm{lCCK}-8$ (Beyotime Institute of Biotechnology, Shanghai, China) reagent per well at $37^{\circ} \mathrm{C}$ for $2 \mathrm{~h}$. Proliferation ability was detected at the selected time points (0, 1, 2 and 3 days following seeding). The optical density was determined at a wavelength of $450 \mathrm{~nm}$.

Cell colony formation assay. The transfected cells $(1,000$ cells/well) were seeded in a 12 -well plate. Following 14 days incubation at $37^{\circ} \mathrm{C}$, cell colonies were counted under a light microscope (magnification, x200) following fixing with $100 \%$ methanol and $0.1 \%$ crystal violet staining for $20 \mathrm{~min}$ at room temperature.

Statistical analysis. All of statistical analyses in the study were performed using SPSS 19.0 software (IBM Corp., Armonk, NY, USA). Data are presented as the mean \pm standard deviation from at least three independent experiments. Difference between two groups was assessed by paired Student's t-test, $\chi^{2}$ test as appropriate and differences between multiple groups was analyzed with one-way analysis of variance with a post hoc Student-Newman-Keuls test. The survival plots were calculated by the Kaplan-Meier method and log-rank test. $\mathrm{P}<0.05$ was considered to indicate a statistically significant difference.

\section{Results}

DUXAP8 expression is upregulated in human glioma tissues. The expression of DUXAP8 in glioma tissues, compared with that in normal tissues, was analyzed. The data revealed that DUXAP8 expression was significantly upregulated in glioma tissues, compared with that in normal tissues (Fig. 1A; P<0.05). Similarly, the data also revealed that DUXAP8 expression was significantly upregulated in glioma cells (U87, U251 and H4 cells), compared with normal NHA cells (Fig. 1B; $\mathrm{P}<0.05$ ). Therefore, these results indicated that DUXAP8 expression is upregulated in human glioma tissues and cells.

Upregulation of DUXAP8 expression is associated with Karnofsky Performance Status (KPS), WHO grade and poor prognosis of glioma. Furthermore, the mean expression of DUXAP8 (2.35 fold) was used as a cut-off value to divide patients into two groups: High DUXAP8 expression and low DUXAP8 expression, with the mean expression of DUXAP8 assigned to the high expression group. The clinicopathological characteristics are presented in Table I. The association of DUXAP8 expression with the clinicopathological characteristics was analyzed using $\chi^{2}$ test. The results indicated that increased DUXAP8 expression was significantly associated with higher KPS $(\mathrm{P}=0.004)$ and advanced WHO grade $(\mathrm{P}=0.001)$ in patients (Table I). However, no significant association with sex, age and tumor size was indicated (Table I; P>0.05). The survival plots were calculated by the Kaplan-Meier methods and log-rank test. The results indicated that increased DUXAP8 expression predicted poor disease-free survival (DFS; log rank test $=12.554 ; \mathrm{P}<0.05$ ) and overall survival (OS; log rank test=13.374; $\mathrm{P}<0.05$ ) rates, compared with reduced DUXAP8 expression groups (Fig. 2A and B). Therefore, these results indicated that DUXAP8 may be a predictor of glioma. 
Table I. Association between DUXAP8 expression and clinical features.

DUXAP8 expression

\begin{tabular}{|c|c|c|c|c|}
\hline & & & & \\
\hline Clinical features & Total cases, $\mathrm{n}=58$ & Low, $n=30$ & High, $n=28$ & $\mathrm{P}$-value \\
\hline Sex & & & & 0.455 \\
\hline Male & 36 & 20 & 16 & \\
\hline Female & 22 & 10 & 12 & \\
\hline Age (years) & & & & 0.300 \\
\hline$\leq 55$ & 31 & 18 & 13 & \\
\hline$>55$ & 27 & 12 & 15 & \\
\hline Tumor size $(\mathrm{cm})$ & & & & 0.771 \\
\hline$\leq 3$ & 32 & 16 & 16 & \\
\hline$>3$ & 26 & 14 & 12 & \\
\hline Tumor location & & & & 0.457 \\
\hline Parenchyma & 40 & 22 & 18 & \\
\hline Ventricle & 18 & 8 & 10 & \\
\hline Karnofsky performance status ${ }^{b}$ & & & & $0.004^{\mathrm{a}}$ \\
\hline$\leq 80$ & 32 & 22 & 10 & \\
\hline$>80$ & 26 & 8 & 18 & \\
\hline WHO grade ${ }^{b}$ & & & & $0.011^{\mathrm{a}}$ \\
\hline $\mathrm{I}-\mathrm{II}$ & 34 & 22 & 12 & \\
\hline III-IV & 24 & 8 & 16 & \\
\hline
\end{tabular}

${ }^{\mathrm{a}} \mathrm{P}<0.05$. ${ }^{\mathrm{b}}$ According to (13). WHO, World Health Organization; DUXAP8, double homeobox A pseudogene 8.
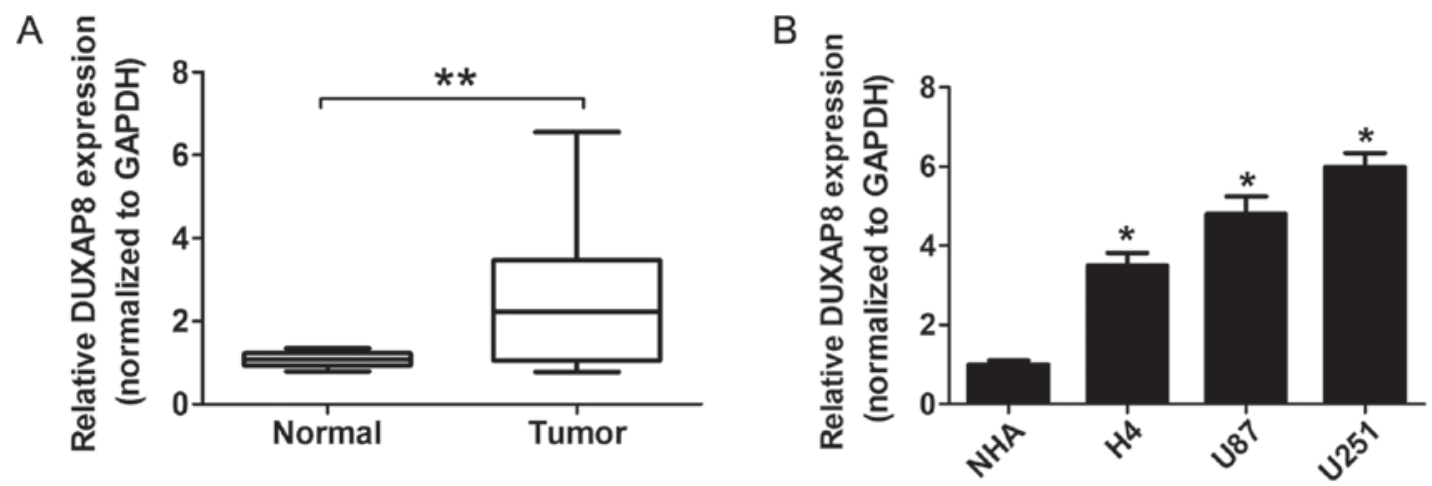

Figure 1. DUXAP8 expression is upregulated in glioma tissues and cells. (A) DUXAP8 expression was detected in 58 paired of human glioma tissues and adjacent normal tissue sample. GAPDH was used as the internal control. (B) DUXAP8 expression was detected in human glioma U87, U251 and H4 cell lines, compared with normal human astrocyte NHA cells. GAPDH was used as the internal control. ${ }^{* *} \mathrm{P}<0.01$ and ${ }^{*} \mathrm{P}<0.05$ vs. NHA cells. DUXAP8, double homeobox A pseudogene 8 .

Knockdown of DUXAP8 expression suppresses proliferation of glioma. To examine the biological effects of DUXAP8 expression, a CCK8 assay and cell colony formation assay were performed following DUXAP8 siRNA treatment. According to knockdown efficiency in U87 and U251 cells, siRNA-1 was selected for the following experiments (Fig. 3A and B). The CCK8-assay results indicated that DUXAP8-knockdown significantly inhibits proliferation, compared with the control groups, in U87 and U251 cells (Fig. 3C and D). Furthermore, the results of colony-formation assays revealed that the clonogenic number is significantly decreased following DUXAP8-knockdown in U87 and U251 cells (Fig. 4A-D). Therefore, these results indicated that DUXAP8-knockdown suppresses the proliferation of glioma.

\section{Discussion}

Pseudogenes were long identified to be non-functional relics littering the genome (15). Previous studies revealed that the involvement of pseudogenes in the pathogenesis and progression of tumors, including the OCT4 pseudogene POU class 5 
A

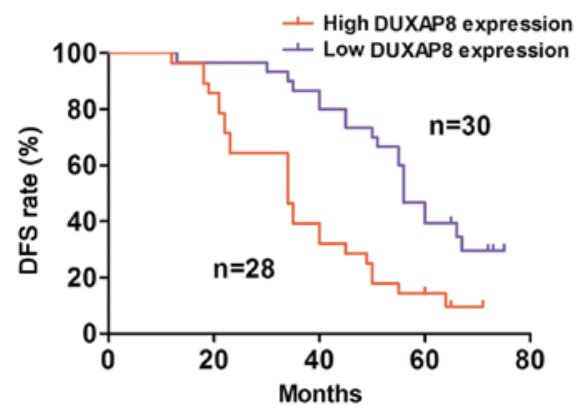

B

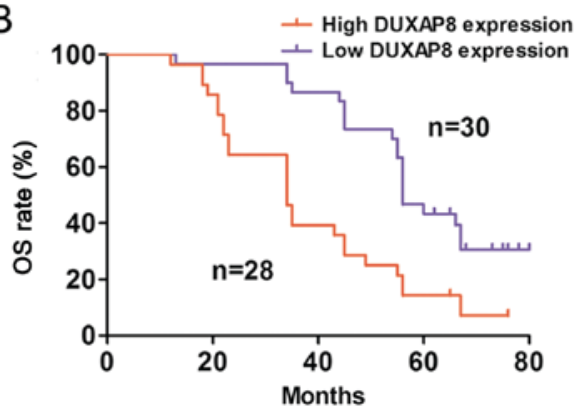

Figure 2. DUXAP8 expression is associated with prognosis of patients with glioma. (A) Increased DUXAP8 expression was associated with poor DFS rate, compared with reduced DUXAP8 expression in patients with glioma. Log rank test, $\mathrm{P}<0.05$. (B) Increased DUXAP8 expression associated with poor OS rate, compared with reduced DUXAP8 expression in patients with glioma. Log rank test, P<0.05. DUXAP8, double homeobox A pseudogene 8; DFS, disease-free survival; OS, overall survival.

A
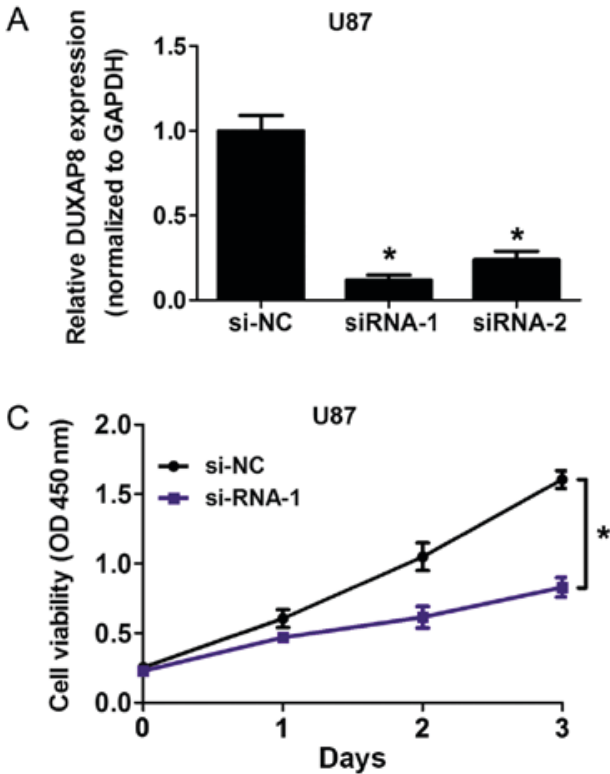

B
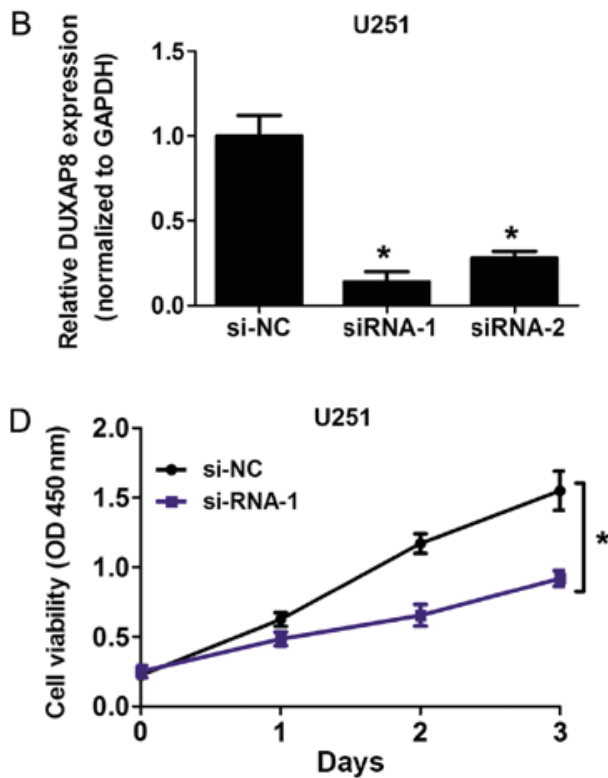

Figure 3. Reduced DUXAP8 expression inhibits proliferation ability. The DUXAP8 expression in (A) U87 cells and (B) U251 cells was detected following transfection of cells with si-NC or si-RNA-1 at $48 \mathrm{~h}$ with CCK8 cell assays. The results of the CCK8 assays following transfection of (C) U87 cells and (D) U251 cells with si-NC or si-RNA-1 at 48 h. "P<0.05, compared with si-NC. DUXAP8, double homeobox A pseudogene 8; si, small interfering; NC, negative control; OD, optical density; Cell-Counting Kit 8.
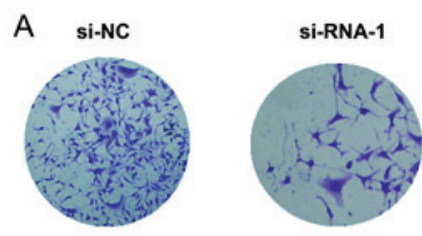

U87
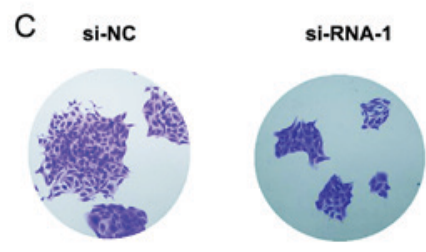

U251
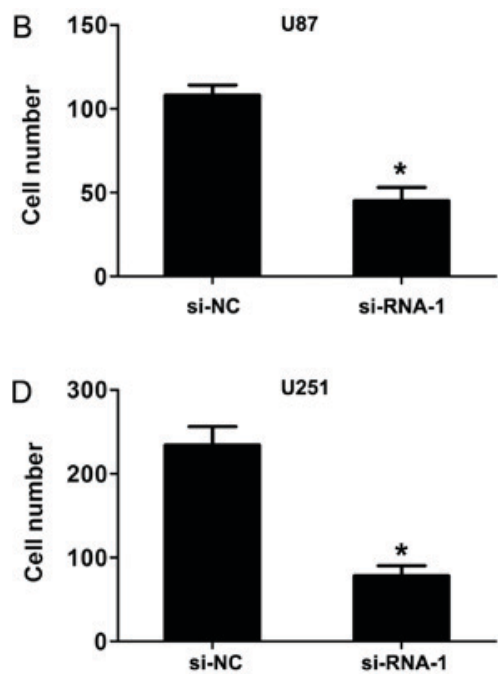

Figure 4. Reduced DUXAP8 expression inhibits cell colony formation ability. The cell colony assays and cell number following transfection of (A) U87 cells and (B) U251 cells with si-NC or si-RNA-1 at 14 days. The cell colony assays and cell number following transfection of (C) U87 cells and (D) U251 cells with si-NC or si-RNA-1 at 14 days. "P<0.05, compared with si-NC. DUXAP8, double homeobox A pseudogene 8; si, small interfering; NC, negative control. 
homeobox 1, amplify and promote an aggressive phenotype in gastric cancer $(8,16)$. SUMO 1 pseudogene 3 was significantly upregulated in gastric cancer and was significantly associated with tumor size, differentiation, lymphatic metastasis and invasion (8). In the present study, it was revealed that DUXAP8 expression was significantly upregulated in glioma tissues and cells, compared with that in normal tissues and NHA cells. Increased DUXAP8 expression was significantly associated with higher KPS scores and advanced WHO grade in patients. Furthermore, the survival curves were calculated by the Kaplan-Meier method and log-rank test. The results of the present study indicated that increased DUXAP8 expression revealed a poor outcome of patients, compared with reduced DUXAP8 expression groups. Therefore, these results indicated that DUXAP8 may be served as a potential predictor of glioma.

Pseudogene DUXAP8 was revealed to regulate tumor progression in numerous tumors. For instance, the pseudogene DUXAP8 promotes non-small-cell lung cancer cell proliferation, and invasion by epigenetically silencing early growth response 1 and Ras homolog family member B (11). Pseudogene DUXAP8 was significantly associated with overall survival time of patients with renal cell carcinoma (RCC), and the knockdown of DUXAP8 may impair RCC cells invasive ability in vitro (17). Increased DUXAP8 promoted cell proliferation and invasion through epigenetically silencing pleckstrin homology domain contain $\mathrm{O} 1$ expression by binding with enhancer of zeste 2 polycomb repressive complex 2 subunit and SUZ12, polycomb repressive complex 2 subunit in gastric cancer cells (12). The present study demonstrated that knockdown of DUXAP8 expression inhibits the proliferation and cell colony formation ability, which indicated that DUXAP8 expression affects the proliferation ability in glioma cells. Therefore, these results indicated that DUXAP8 is involved in biological functions of glioma.

In conclusion, the results of the present study first revealed that DUXAP8 expression is significantly upregulated in glioma tissues and cells. Increased DUXAP8 expression predicted a poor survival rate. Additionally, DUXAP8-knockdown significantly inhibited proliferation in glioma. These results indicated for the first time, to the best of our knowledge, that pseudogene DUXAP8 may be a potential biomarker and target of tumor treatment in glioma. Future studies should investigate the molecular mechanism for DUXAP8 in glioma progression.

\section{Acknowledgements}

Not applicable.

\section{Funding}

Not applicable.

\section{Availability of data and materials}

The datasets used and/or analyzed during the present study are available from the corresponding author on reasonable request.

\section{Authors' contributions}

$\mathrm{XZ}, \mathrm{SH}, \mathrm{MW}$ and $\mathrm{CW}$ conceived and designed the study, and drafted the manuscript. DX, XZ, SH, and MW collected, analyzed and interpreted the experiment data, and revised the manuscript critically for important intellectual content. All authors read and approved the final manuscript.

\section{Ethics approval and consent to participate}

The study was approved by the Ethics Committee of The Second Hospital of Shandong University (Jinan, China). Written informed consent was obtained from all patients in the study.

\section{Patient consent for publication}

Not applicable.

\section{Competing interests}

The authors declare that they have no competing interests.

\section{References}

1. Taylor LP: Diagnosis, treatment, and prognosis of glioma: Five new things. Neurology 75 (18 Suppl 1): S28-S32, 2010.

2. Lenting K, Verhaak R, Ter Laan M, Wesseling P and Leenders W: Glioma: Experimental models and reality. Acta Neuropathol 133: 263-282, 2017.

3. Hirst TC, Vesterinen HM, Conlin S, Egan KJ, Antonic A, Lawson McLean A, Macleod MR, Grant R, Brennan PM, Sena ES and Whittle IR: A systematic review and meta-analysis of gene therapy in animal models of cerebral glioma: Why did promise not translate to human therapy? Evid Based Preclin Med 1: e00006, 2014.

4. Wei Y, Chang Z, Wu C, Zhu Y, Li K and Xu Y: Identification of potential cancer-related pseudogenes in lung adenocarcinoma based on ceRNA hypothesis. Oncotarget 8: 59036-59047, 2017.

5. Xiao-Jie L, Ai-Mei G, Li-Juan J and Jiang X: Pseudogene in cancer: Real functions and promising signature. J Med Gen 52: 17-24, 2015.

6. Harrison PM, Hegyi H, Balasubramanian S, Luscombe NM, Bertone P, Echols N, Johnson T and Gerstein M: Molecular fossils in the human genome: Identification and analysis of the pseudogenes in chromosomes 21 and 22. Genome Res 12: 272-280, 2002

7. Jingsi T, Mingyao Y and Ying L: Functional roles of pseudogenes in cancers. Yi Chuan 37: 8-16, 2015.

8. Mei D, Song H, Wang K, Lou Y, Sun W, Liu Z, Ding X and Guo J: Up-regulation of SUMO1 pseudogene 3 (SUMO1P3) in gastric cancer and its clinical association. Med Oncol 30: 709, 2013.

9. Zhan Y, Liu Y, Wang C, Lin J, Chen M, Chen X, Zhuang C, Liu L, $\mathrm{Xu} \mathrm{W}, \mathrm{Zhou} \mathrm{Q}$, et al: Increased expression of SUMO1P3 predicts poor prognosis and promotes tumor growth and metastasis in bladder cancer. Oncotarget 7: 16038-16048, 2016.

10. Huang W, Li N, Hu J and Wang L: Inhibitory effect of RNA-mediated knockdown of zinc finger protein 91 pseudogene on pancreatic cancer cell growth and invasion. Oncol Lett 12: 1343-1348, 2016.

11. Sun M, Nie FQ, Zang C, Wang Y, Hou J, Wei C, Li W, He X and Lu KH: The Pseudogene DUXAP8 promotes non-small-cell lung cancer cell proliferation and invasion by epigenetically silencing EGR1 and RHOB. Mol Ther 25: 739-751, 2017.

12. Ma HW, Xie M, Sun M, Chen TY, Jin RR, Ma TS, Chen QN, Zhang EB, He XZ, De W and Zhang ZH: The pseudogene derived long noncoding RNA DUXAP8 promotes gastric cancer cell proliferation and migration via epigenetically silencing PLEKHO1 expression. Oncotarget 8: 52211-52224, 2017. 
13. Louis DN, Ohgaki H, Wiestler OD, Cavenee WK, Burger PC, Jouvet A, Scheithauer BW and Kleihues P: The 2007 WHO classification of tumours of the central nervous system. Acta Neuropathol 114: 97-109, 2007.

14. Livak KJ and Schmittgen TD: Analysis of relative gene expression data using real-time quantitative PCR and the 2(-Delta Delta C(T)) method. Methods 25: 402-408, 2001.

15. Pink RC, Wicks K, Caley DP, Punch EK, Jacobs L and Carter DR Pseudogenes: Pseudo-functional or key regulators in health and disease? RNA 17: 792-798, 2011.
16. Hayashi H, Arao T, Togashi Y, Kato H, Fujita Y, De Velasco MA, Kimura H, Matsumoto K, Tanaka K, Okamoto I, et al: The OCT4 pseudogene POU5F1B is amplified and promotes an aggressive phenotype in gastric cancer. Oncogene 34: 199-208, 2015.

17. $\mathrm{Xu} \mathrm{X}, \mathrm{Xu} \mathrm{Y}$, Shi C, Wang B, Yu X, Zou Y and $\mathrm{Hu} \mathrm{T}$ : A genome-wide comprehensively analyses of long noncoding RNA profiling and metastasis associated lncRNAs in renal cell carcinoma. Oncotarget 8: 87773-87781, 2017. 